\title{
Über das Sprechen und das Schweigen in der Medizin
}

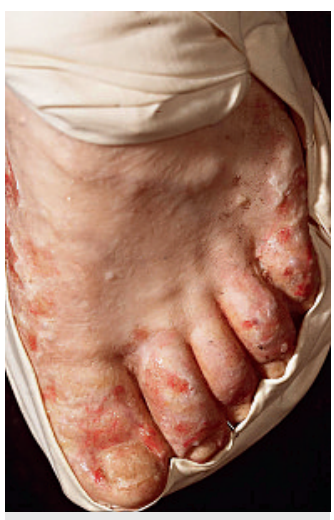

Moulage eines Fusses mit Hautmykose (Moulagenmuseum Universität und UniversitätsSpital Zürich).

\section{Mit diesem Thema befasst sich auch der} Beitrag auf Seite 640 .

1 Hierzu aktuell: Tel Aviver Jahrbuch für Deutsche Geschichte 2011; 39 (Thema: Trauma und Holocaust).

2 Boll-Palievskaya D. Migration und Gesundheit: Andere Sitten andere Diagnosen. Deutsches Ärzteblatt. 2009;106 A-1929, B-1657, C-1625.

* PD rer. soc. Eberhard Wolff ist Kulturwissenschaftler, Medizinhistoriker und Mitglied der Redaktion Medizingeschichte der Schweizerischen Ärztezeitung.
Was, denken Sie, sind typische Tabu-Krankheiten? Ein Blick hinüber zum Liegestuhl-Nachbar am Kanarenpool gab mir vor ein paar Wochen eine mögliche Antwort. «Die Tabu-Krankheiten der Deutschen» betitelte «Bild» seine aktuelle Serie und klärte kurz und knapp über Herpes, Genitalwarzen, Hämorrhoiden, Hautpilze, Blähungen, Parasiten, Inkontinenz, Mundgeruch, Schuppen oder die Überproduktion von Schweiss und Ohrenschmalz auf. Zugegeben: Über die Repräsentativität dieser Auswahl lässt sich streiten. Mit ihrem Schwerpunkt auf Ekel, Körperabsonderungen und Intimzonen trifft sie aber schon ein typisches Feld von Krankheiten, über die man heute nicht so gerne öffentlich redet, ausser vielleicht in Internetforen und Nachmittagstalkshows. Zu denken gibt auch, wie oft Formen von begrenzter Körperkontrolle offensichtlich als «peinlich» empfunden werden. Und mit Fusspilz und Kopfläusen ist auch noch der Komplex derjenigen Infektionskrankheiten vertreten, bei denen die moralische Aura hygienischer Nachlässigkeit mitschwingt, selbst wenn dies medizinisch gar nicht gerechtfertigt sein mag.

Am Rande zählte «Bild» auch Depressionen zu den «Tabu-Krankheiten». Gesellschaftliches NichtFunktionieren oder «aus der Norm fallen» wird ebenfalls nicht gerne an die grosse Glocke gehängt. Der Suizid des Deutschen Nationalgoalies Robert Enke machte dies 2009 ganz plötzlich zum grossen Thema.

Vor fünfzig oder hundert Jahren hätte die Liste sicher anders ausgesehen. Daniel Kauz beschreibt in seiner Geschichte der Krebsliga Schweiz unter dem Titel «Vom Tabu zum Thema?», wie sehr der Krebs eine tabuisierte Krankheit war - im landläufigen, nicht streng wissenschaftlichen Verständnis des Begriffs «Tabu» (siehe die plastischen Beispiele im Artikel auf S. 640). Man wollte nicht darüber sprechen, unter anderem weil die Krankheit damals nicht nur häufig fatal, sondern durch ihre wenig erklärliche Ätiologie und ihren Verlauf unheimlich erschien und zum Teil bis heute erscheint.

Interessant ist an der Beschreibung von Kauz, wie die «Tabuisierung» der Krankheit vom professionellen Medizinsystem damals auch aktiv mitbetrieben wurde. Etwa mit der «heiligen Lüge» gegenüber Patienten, die mit der Diagnose nicht zurechtzukommen schienen oder Angst hatten, von ihrer Umwelt als Krebskranke «schief» angesehen zu werden. Gedacht war das Nichtsprechen als ein Entgegenkommen gegenüber den Betroffenen, aber mit der Folge eines «Kreislaufs von Verheimlichung und Misstrauen».
Wenn es sich die Krebsliga später im Gegenteil zur Aufgabe gemacht hat, gegen dieses Tabu anzuarbeiten, dann geschah dies ebenfalls mit dem Ziel, Patientenbedürfnissen entgegenzukommen, nur eben anderen: dass sich Menschen offen über ihre Krankheit austauschen und ihr weiteres Leben aktiv in die Hand nehmen können. Heute wird viel offener und öffentlicher über Krebs gesprochen.

Nicht nur in der Krebsliga weiss man, dass in anderen Kulturen, etwa von Migranten, das Nichtsprechen über bestimmte Krankheiten, sei es nun Krebs oder seien es psychische Erkrankungen, wesentlich häufiger ist, dass hier Respekt und Vorsicht angebracht sind und kommunikatives Einfühlungsvermögen hilft. Das bestätigt auch das Institut für Migration, Kultur und Gesundheit «AMIKO» in Freiburg/Br.

Sind Tabus dazu da, in jedem Fall gebrochen zu werden? Eine ganze Generation von Holocaust-Überlebenden stand vor dem kaum zu bewältigenden Problem, mit dem Erlebten irgendwie umgehen zu müssen [1]. Viele entschieden sich dafür, nicht darüber zu sprechen, das Thema zu tabuisieren. Für viele von ihnen war es eine Überlebensstrategie - und vielleicht nicht die schlechteste. Darüber mögen Psychologen streiten. Oder, wie es der Chefredaktor dieser Zeitschrift Bruno Kesseli auf der Basis seiner Erfahrungen mit HIV-Patienten ausdrückt: «Tabuisierung kann in gewissen Kreisen auch sinnvoll sein (leider).»

Tabus im strengen Sinne des Begriffs sind einfach gesagt nicht hinterfragte gesellschaftliche Sprechoder Handlungsverbote, die es in allen Gesellschaften gibt (Stichwort: Political correctness). Sie sind häufig mit existentiellen Themen wie dem Tod verbunden. In ihnen spiegelt sich die Kultur einer Gesellschaft, und Tabus können sich mit ihr verändern. Sie können repressiv wirken, aber auch Schutz anbieten.

«Vom Tabu zum Thema?» Mir fällt bei diesem Buchtitel das Fragezeichen auf. Für mich stellen sich auch vor allem Fragen: Wer schweigt über eine Krankheit: Betroffene, Behandelnde, das Umfeld? Aus welchen Gründen, aus Selbstschutz, Rücksichtnahme oder Konvention? Welche Patientenbedürfnisse sollen wie respektiert werden? Kann man es den Patienten immer recht machen? Sigmund Freud soll seinem Arzt nach Eröffnen der Diagnose Zungenkrebs gesagt haben: «Wer hat Ihnen erlaubt, mir davon zu erzählen?» [2].

Eberhard Wolff* 